\title{
Evaluation of the prevalence and severity of xerophthalmia in head and neck cancer patients undergoing curative radiotherapy
}

\author{
Sowmya V' , Dipika Jayachander ${ }^{2}$, Vijna Kamath' ${ }^{1}$, Mithun SK Rao ${ }^{3}$, \\ Mohammed Raees Tonse ${ }^{2}$, Manjeshwar Shrinath Baliga ${ }^{*}$ \\ 'Department of Ophthalmology, Father Muller Medical College, Karnataka, India; \\ ${ }^{2}$ Department of Radiation Oncology, Father Muller Medical College, Karnataka, \\ India; ${ }^{3}$ Father Muller Medical College, Kankanady, Mangalore, Karnataka, India \\ 575002; ${ }^{4}$ Father Muller Research Centre, Kankanady, Mangalore, Karnataka, India \\ 575002
}

\begin{abstract}
Background: The study objective was to assess the development of xerophthalmia [dry eye syndrome (DES) or keratoconjunctivitis sicca] in head and neck cancer patients undergoing radiotherapy.

Methods: Twenty two head and neck cancer patients requiring more than 60 Gy of curative radiotherapy/chemoradiotherapy and ten patients requiring radiotherapy/chemoradiotherapy for treating cancers in the non head and neck regions (like breast, oesophagus, prostate, cervix and rectal cancers) were also enrolled in the study. The development of $D E S$ was studied at the beginning (day 0, before the start of radiotherapy) at day 21 (after completion of $30 \mathrm{~Gy}$ ) and on completion of the treatment (>60 Gy). As a comparative cohort, people with non head and neck cancer needing curative radiotherapy were also evaluated for comparison.

Results: There was no difference in degree of DES between the Head and Neck cancer cohorts and non head and neck group at the beginning of treatment. However there was a statistically significant difference $(p<0.001)$ between the two groups at both mid and end of RT time point. Inter comparison between the various time points in the head and neck cancer group showed that the incidence of DES increased with the radiation exposure and was significant ( $p r e$ to mid $p<0.001$; and mid to end $p<0.005)$. A negative $(r=-0.262)$ correlation was seen between DES and distance.

Conclusions: The study showed that lesser the distance from the epicenter of the radiation to the orbital rim more was the severity of DES.
\end{abstract}

Key words: xerophthalmia, dry eye syndrome, keratoconjunctivitis sicca, ionizing radiation, Head and neck cancer

\section{Introduction}

lonising radiation, which is an important modality in the treatment of head and neck cancers, is associated with various side effects like treatment induced oral mucositis, xerostomia, and dysphagia ${ }^{1}$. In addition to these, xerophthalmia [also known as dry eye syndrome (DES) or keratoconjunctivitis sicca], which develops

Correspondence: Dr Sowmya V., Father Muller Hospital Road, Kankanady, Mangalore, Karnataka, India 575002.

E-mail: drsowmyav@yahoo.com 
when the field of radiation includes the orbit and the tissues adjacent to the eye like adnexa, the lacrimal system (including the major and accessory lacrimal glands, lacrimal canaliculi, lacrimal sac, and nasolacrimal duct), the ocular surface (including the cornea and conjunctiva), the motor nerves, the eyelids, the meibomian glands and associated sensory organs is also a commonly observed side effect. ${ }^{2-5}$ Xerophthalmia occurs as a result of damage to glands within the eyelid, decreased conjunctival mucous production and reduced lacrimal gland secretion. The functional effects of all these alterations are conjunctival inflammation, chemosis, tear film instability and a resultant dry eye sensation, which generally subside but on occasion, be persistent. ${ }^{6}$
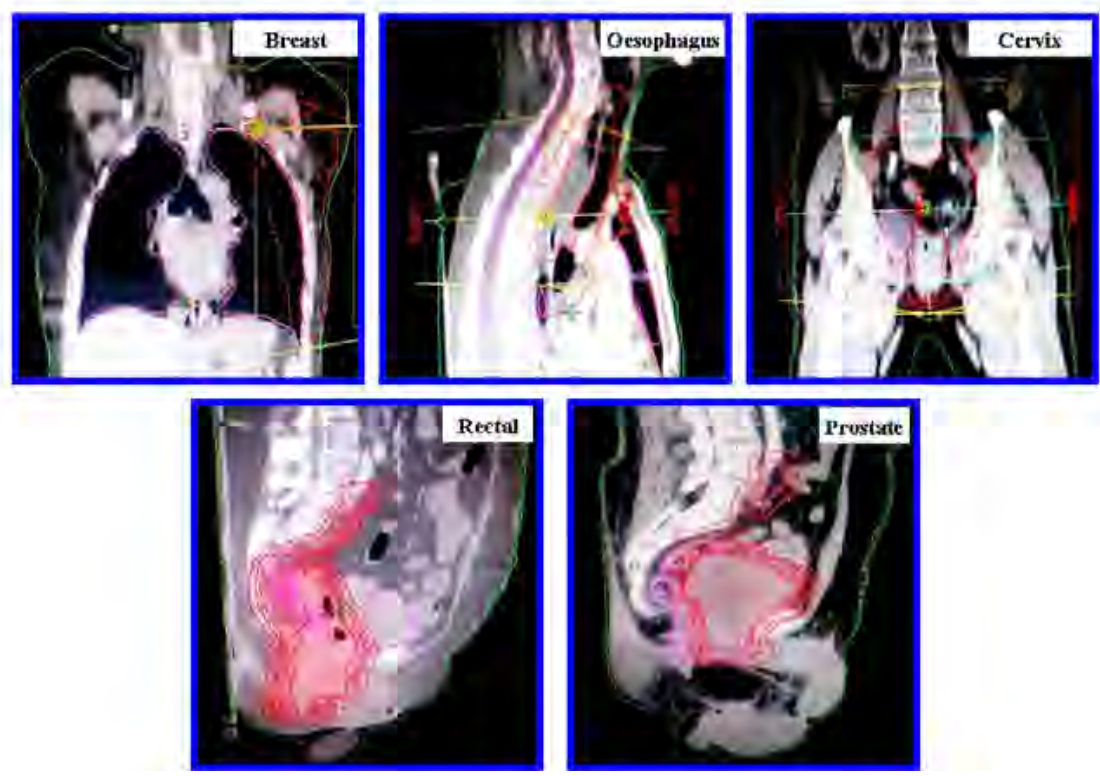

Fig 1a. Film pictures depicting the RT fields planned in various cancers. (Design: ...)

The incidence of DES is proportionate to the total dose of radiotherapy and results from the cytostatic effects of radiation on the cells with high turnover and to specific alterations in cell type and function. ${ }^{5}$ These effects are dose-dependent and the incidence of dry eye increases steeply at doses $>40 \mathrm{~Gy}$ and observations have indicated the long term effects post irradiation. ${ }^{7,8} \mathrm{DES}$ increases osmolarity of the tear film and inflammation of ocular surface. ${ }^{8}$ Reports indicate that the increase in the stratification of conjunctival epithelia and reduction in goblet cell numbers contributes to a dry eye following radiotherapy ${ }^{6}$, and also that loss of serous acinar cells from the lacrimal gland contributes towards the DES. ${ }^{9}$

Clinically, the common symptoms of severe DES include dryness, stinging, burning, irritation, itching, scratchy eye, tired eye, photophobia, pain, redness, a foreign body sensation, mucous discharge, excessive tearing, blurry vision in the 
early stages, and ulceration, vascularization, opacification, perforation and vision loss in advanced stages. ${ }^{6,8}$ Although mild to moderate DES is often successfully manageable with supportive therapy, severe DES can result in ulceration and/or infection of the cornea, thickening of the corneal surface, corneal erosion, punctate keratopathy, epithelial defects, corneal abrasions, ulceration, neovascularization, scarring, thinning, perforation, and corneal necrosis, leading to irreversible corneal opacity with compromised vision, severe pain, and eye loss. ${ }^{10}$
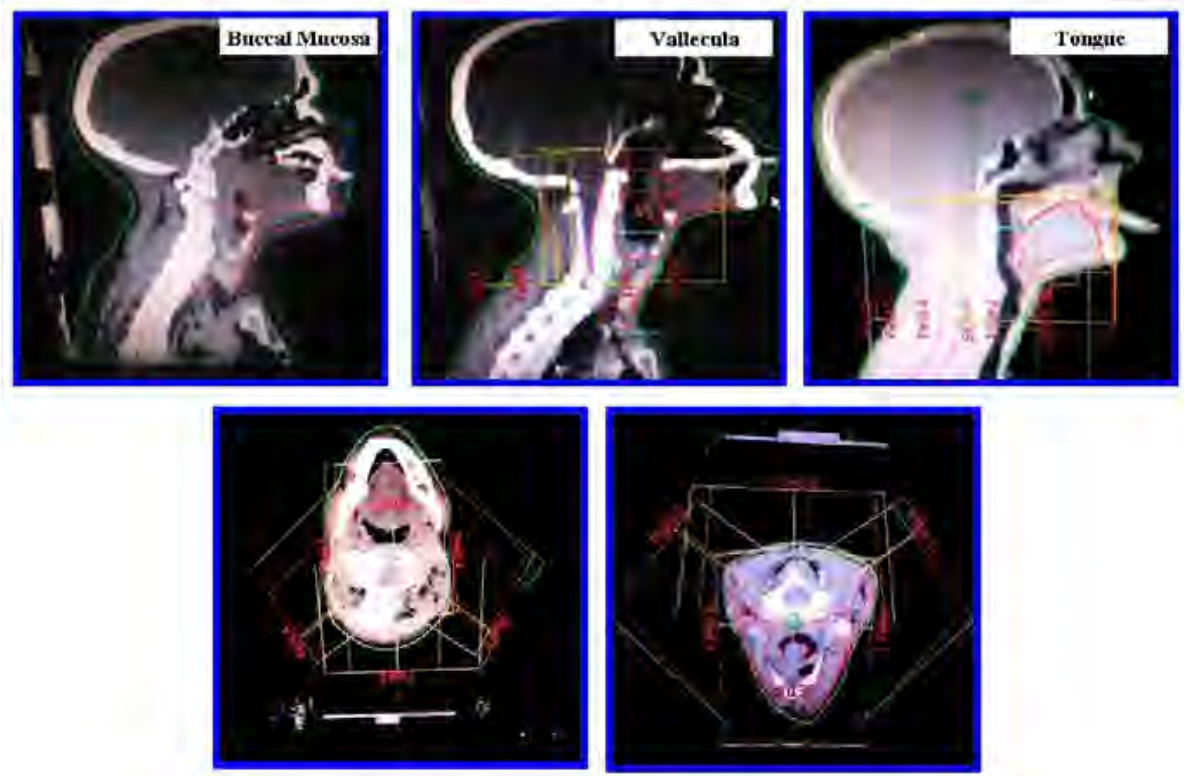

Fig 1 b. Film pictures depicting the RT fields planned in various head and neck cancers. (Design: ...)

DES is classified in accordance to the guidelines set by The International Dry Eye Workshop and modified by the Delphi Panel Report in to four groups as mild, moderate, severe, and severe and/or disabling (Table 1). ${ }^{10-12}$ Depending on the manifestation, the radiation-induced DES are classified as either early (acute) or late (delayed) effects and is dependent on the dose of radiation incurred. The early effects develop during the course of RT or shortly after completing RT (about 2-3 weeks), while the late effects manifest months to years after completing the therapeutic radiotherapy. ${ }^{10-12}$

When compared to other radiation-induced ill effects, DES is sparsely reported in patients being treated for their head and neck cancers. However reports do indicate that DES increases the morbidity and that this at times can severely affect the patient's quality of life and to increase medical hospitalization and treatment costs. ${ }^{8}$ The present study was investigated to assess the incidence of dry eye syndrome during external beam radiotherapy for extra cranial head and neck tumours, and correlate its severity with distance from the epicenter of the field of radiation. 


\section{Materials and methods}

This was a single-centre; investigator masked prospective study and was conducted from April to June 2013 in the Department of Radiation Oncology at Father Muller Medical College Hospital, Mangalore India. The subjects comprised of histopathologically confirmed adult patients of head and neck cancer scheduled to receive chemoradiotherapy for curative purpose. Patients with recurrence of cancer in the oral cavity were eligible. Exclusion criteria included patients less than 18 years of age; patients who were pregnant, patients who had oral surgery within the previous 6 weeks, received chemotherapy or radiation treatment previously to the head and neck region; patients with co morbid conditions like poorly controlled diabetes mellitus, hypertension, schizophrenia, bipolar disorders, severe depression.

The study also included a second group of patients requiring curative radiotherapy for non head and neck cancers (like for breast, oesophagus, prostate, cervix and rectal cancers) as control group. The study aimed to recruit subjects into two groups giving $80 \%$ power to detect an estimated $50 \%$ difference between the two groups. It was required to calculate the difference in proportions between two groups for which nMasterTM 1.0 software was used. Each group was calculated and it was observed that a minimum of eight subjects were required to achieve statistical results. The study was approved by the Institutional Ethical Committee. All procedures followed were in accordance with the ethical standards of the responsible committee on human experimentation (institutional and national) and with the Helsinki Declaration of 1975, as revised in 2008.

\section{Patient recruitment:}

During the first visit, one of the investigators (DJ/ MRT/MSR) introduced the purpose of the study to eligible patients and their caregivers in English or in their mother tongue (Kannada, Tulu and Malayalam). The subjects were also informed that they had the right to withdraw from the study at any time during the course of the study and that their non-willingness to be a part of the study will not deprive them of the necessary treatment. Written informed consent was collected from all willing patients satisfying the inclusion criteria.

\section{Radiation therapy treatment}

All patients who participated in this study received external irradiation from a linear accelerator (Varian) at an average energy level of $6 \mathrm{KV}$ MV using the intensity-modulated radiation therapy (IMRT) technique with suitable field planning as depicted in Figure 1a,b. All planned fields were treated every day with no more than one fraction of $2 \mathrm{~Gy}$ per day, five times a week without any intended gaps for a planned target dose of $>60$ Gy (six to seven consecutive weeks) for head and neck cancers and 50 to $70 \mathrm{~Gy}$ for non head and neck cancers depending on the organ dependent protocols (breast and cervix $50 \mathrm{~Gy}$, rectal, prostate and oesophagus > $60 \mathrm{~Gy}$ ). Whenever chemo-irradiation was planned, cisplatin infusion was administered on a weekly basis before exposure to the first weekly radiation. 


\section{Patient Evaluation}

The patients were assessed for the radiation-induced DES by a trained ophthalmologist (SV) unaware of the stage and dose of radiation delivered by the patients. The assessment was undertaken at the beginning (day 0 , before the start of radiotherapy) at day 21 (after completion of $30 \mathrm{~Gy}$ ) and on completion of the treatment. The DES was categorized as mild, moderate, severe, and severe and/or disabling in accordance to the guidelines stipulated by the International Dry Eye Workshop and adopted and modified the Delphi Panel Report, 2007 (Table 2)..$^{10,12}$ On every investigation, the investigator considered the score for the worst toxicity in the treatment field. Patients who discontinued treatment or succumbed to cancer were not considered for the subsequent evaluation. As only one masked researcher evaluated all patients, calibration of assessors was not required.

\section{Tests performed:}

The following assays were performed on all the cases and controls before starting of RT, after 3 weeks of RT and at the end of RT completion: 1) Schirmer I (without local anesthesia), which measures basic secretion of the lacrimal gland, 2) Schirmer II (with local anaesthesia) test, which measures reflex secretion of the lacrimal gland 3) Tear-film break up time (TFBUT), which measures the time of onset of a random appearance of the first black spots on the cornea after one single palpebral (eyelid blinking) closure under standardized conditions which assess goblet cell function, 4) corneal and conjunctival fluorescein staining and 5) Rose Bengal staining of the ocular surface in accordance to the standard ophthalmological investigations in the department of ophthalmology. The patients were first also interviewed to survey the frequency of occurrence of various dry eye symptoms including dryness, grittiness, redness, excess tearing or watery eyes, sensitivity (to smoke, wind, air conditioning) and soreness. Response categories used for analyses included never, seldom (two to three times per week), often (four to five times per week), and always (everyday).

\section{Statistical Analysis:}

The significance of difference of the values between the study groups (control and test) was evaluated by Mann-Whitney $U$ test. To observe for the progression of dry eye syndrome in the test through the treatment period (before RT, in the middle and post RT) was done using the Friedman test and inter time point comparison was done using Wilcoxon signed rank test. The correlation between the degree of dry eye severity with age, gender and distance was done using Pearson's correlation. A value of $p<0.05$ was considered statistically significant.

\section{Results}

The details of age, gender, tumor site and staging, and radiation dose are represented in Table 2. During the study period we could enrol 10 patients requiring radiation treatment to eradicate their cancer in the non head and neck regions like breast, oesophagus, prostate, cervix and rectal cancers, and 22 patients requiring 
radiotherapy for head and neck region. The first group served as the control while the second was the test or experimental cohort. In the control group all the ten patients completed the study and were available for evaluation at all the three time points while in the test group two out of the 22 patients discontinued the treatment (Figure 2).

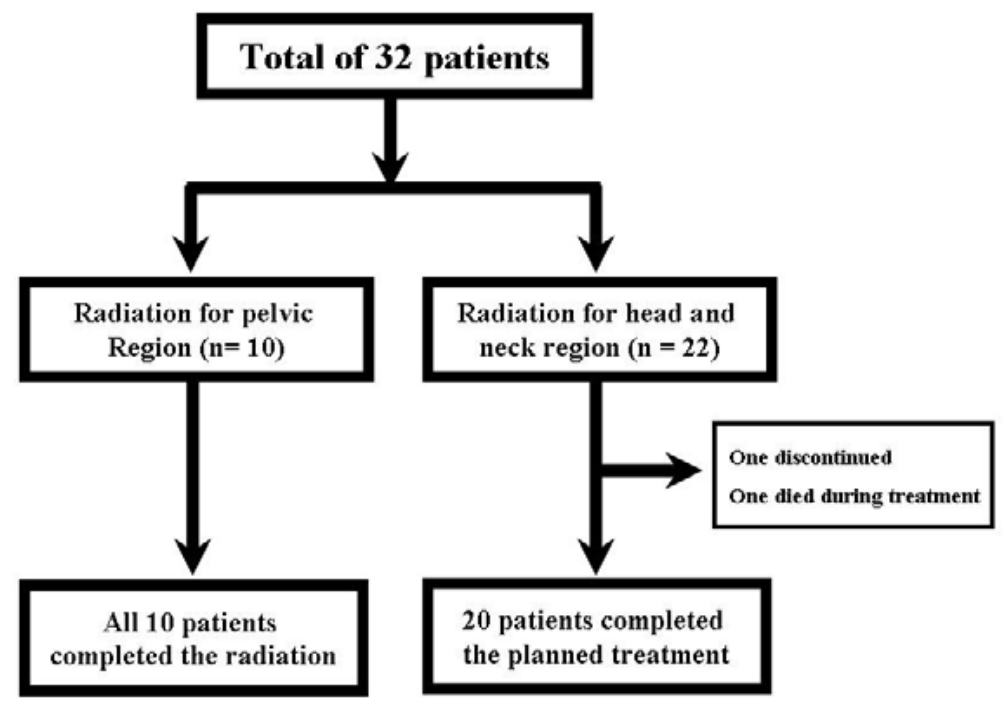

Fig 2. Flow chart of the study population in the two groups

It was observed that before start of radiotherapy majority of the head and neck cancer patients (19 of the 22 patients) did not have any signs of symptoms of DES, while three had level 1 DES (13.64\%). After 3 weeks of RT only 2 (9.1\%) patients did not show any signs or symptoms of DES whereas 9 (40.9\%) showed level1 DES, 9 (40.9\%) showed level 2 DES and 2 (9.1\%) showed level 3 DES. After completion of radiotherapy i.e., after 6-7 weeks of radiotherapy $2(10 \%)$ patients remained asymptomatic whereas 3(15\%) showed level 1 DES, 10 (50\%) showed level 2 DES and 5 (25\%) developed level 3 DES. Over all $90 \%$ of the cases developed DES at the end of the radiotherapy to head and neck region (Figure 3, 4).

The statistical analysis to ascertain the difference of the values between the study groups (control and test) done by Mann-Whitney $U$ test showed no difference at the beginning while a statically significant $(p<0.001)$ difference between the two groups at both Mid and end of RT time point. The Friedman's test used to assess the progression of dry eye severity in the test through the treatment period (before art, in the middle and post RT) showed a significant difference between the pre to mid ( $p<0.001)$ and mid to end of RT ( $p<0.005)$. The Wilcoxon signed rank test also showed significant difference in all the comparisons $(p<0.001$ to 0.0001$)$. The Pearson's correlation analysis showed no significant correlation between the degree of dry eye severity with both age and gender; while a negative $(r=-0.262)$ 
correlation was observed with the distance indicating that lesser the distance from the epicenter of the tumor radiation to the orbital rim more was the dry eye severity.
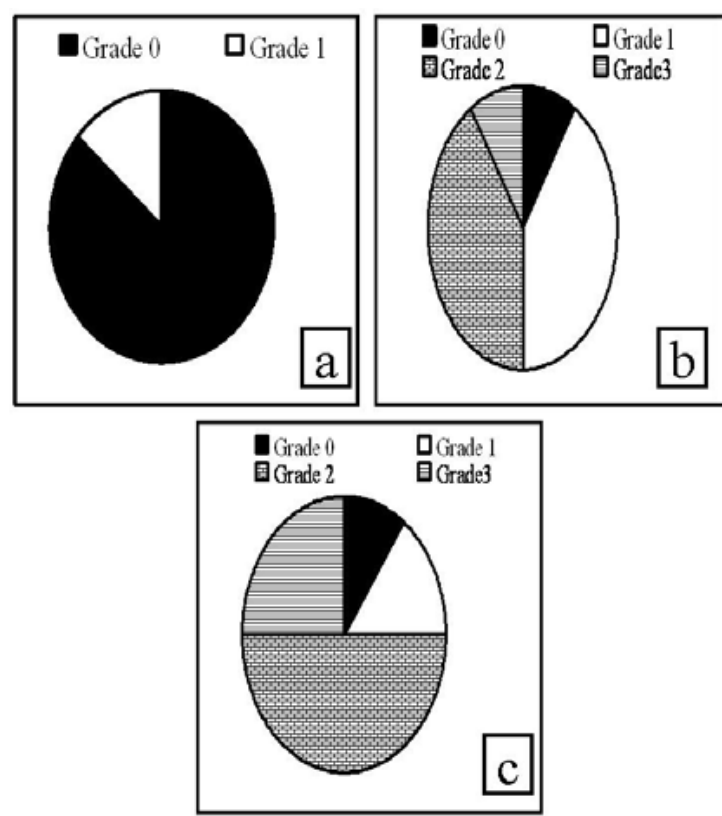

Fig 3. Incidence of different grades of dry eyes syndrome in pre (a), mid (b) and end (c) of the radiation therapy for head and neck cancers.

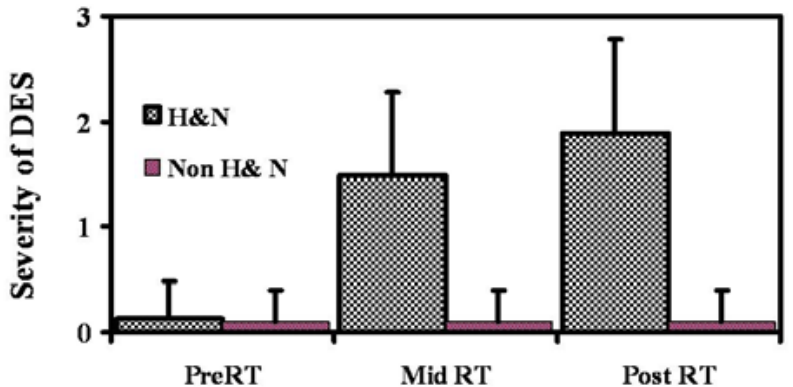

Evaluation Time points
Fig 4. The mean value of the dry eyes syndrome in the pre, mid and post radiation therapy for head and neck.

An analysis stratifying the whole study group based on the distance between the epicenter of the treatment point with orbital margin $(<6.5 \mathrm{~cm}$ and $>6.5 \mathrm{~cm})$ with the severity of DES at the end of the treatment showed that there was a statistically significant difference $(p=0.037)$ between the mean values of the two stratified groups (mean of $2.5 \pm 0.5$ for $<6.5 \mathrm{~cm}$ group vs $1.56 \pm 0.86$ for $>6.5 \mathrm{~cm}$ group Figure 5). 


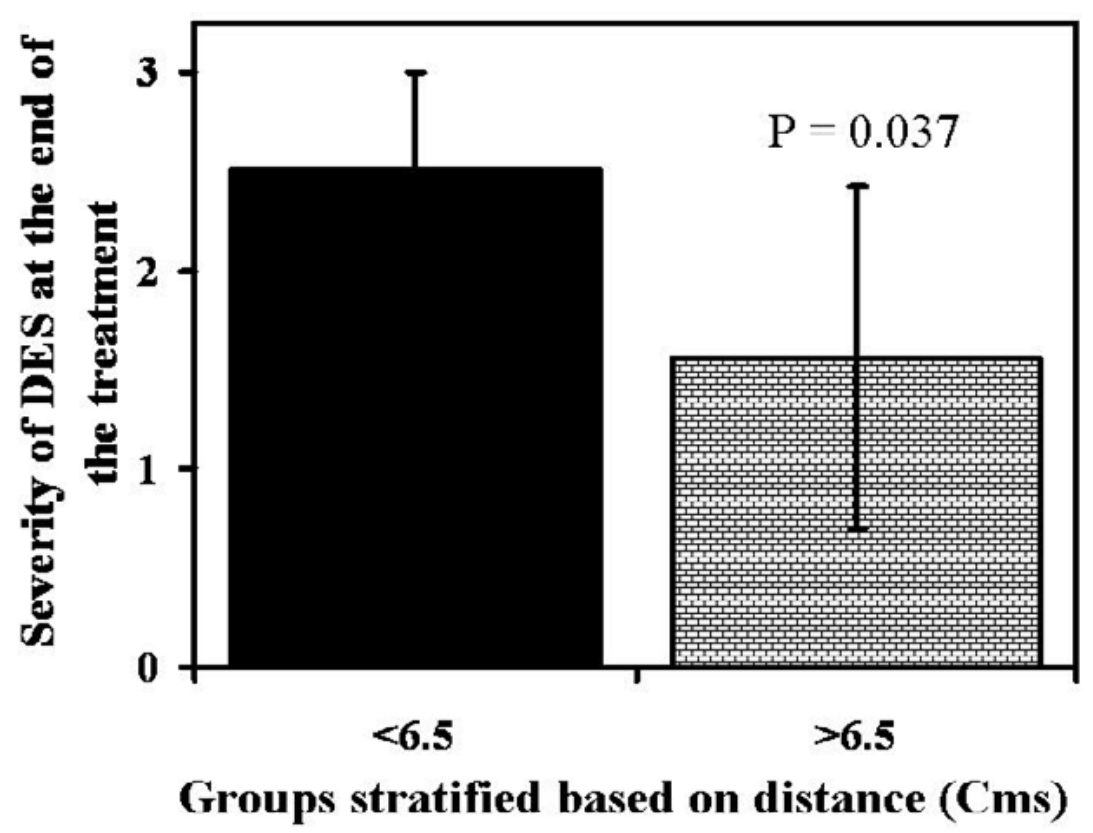

Fig 5. Difference in the degree of DES between the groups where the distance between the epicenter of the treatment point with orbital margin was less than $6.5 \mathrm{~cm}$ (solid) and more than 6.5 $\mathrm{cm}$ (bricks) at the end of the treatment

\section{Discussion}

Xerophthalmia or dry eye syndrome (DES) is an important side effect of therapeutic radiation to head and neck cancer and compromises the quality of life of the patient. However when compared to the other side effects seen in curative radiotherapy for Head and Neck Cancers there are limited data on DES especially during the course of the treatment. Exposure to ionising radiation affects proliferative cells and thereby impairs the optimal functioning of the lacrimal gland. This consequentially initiates the acute and long-term ophthalmic consequences for the patient. Besides the discomfort, the dryness of the eye may cause severe visual impairment due to the resulting corneal damage. . $^{10,12}$

In this study it was observed that all evaluable patients receiving radiation treatment for head and neck cancers developed DES over the course of the treatment and that the severity was proportionate to the exposure dose and inversely with the distance between the orbital margin and field of radiation. According to the authors this is the first study that has evaluated the DES during the course of the treatment. Previous reports on radiation-induced xerophthalmia have all been as a late effect of radiation ${ }^{4,7,13}$. These reports also indicate that exposure to $30-45 \mathrm{~Gy}$ of radiation causes the appearance of DES in 4-11 years, while those above $57 \mathrm{~Gy}$ causes the appearance of corneal vascularization and opacification usually within 
9-10 months post completion of the stipulated therapy. $4,7,13$

The tear film consists of three layers: a superficial lipid layer, derived from meibomian and Zeis gland secretions, which helps retard evaporation; a middle aqueous layer, produced by the major and accessory lacrimal glands: and a deep mucinous layer secreted by goblet cells, which serves to wet the relatively hydrophobic cornea and conjunctival epithelium., ${ }^{2,3}$ Deficiency of any of the three components may result in loss of tear-film stability. ${ }^{2,3}$ Changes in quality and quantity of tear production lead to impairment of the dynamic stability of the tear film resulting in chronic dry eye and in turn damaging the conjunctival and cornea 1 epithelium., ${ }^{2,3}$ Of all the tests performed, it was also observed that the Schirmer test was more affected than other staining tests indicating that the lacrimal gland are affected the most during these interventions.

\section{Conclusion}

The present study for the first time has made an attempt to assess the incidence of DES during the course of curative radiotherapy for head and neck cancers. The results suggest that DES was observed in all the patients available for the estimation at all three time points. It was clearly observed that the incidence and severity of DES was inversely proportional to the distance, indicating that lesser the distance from the center of the tumor radiation to the orbital rim, more was the dry eye severity. It is also suggestive that adequate shielding the anterior structures including cornea, conjunctiva and lacrimal gland should be followed to minimize DES. Future studies are planned to understand the role of lacrimal shield in reducing the adverse effects.

\section{Acknowledgements:}

The authors would like to express thanks to all participants of this study for their kind cooperation. They are also grateful to Dr MS Vidyasagar Former Head of Radiation Oncology, Father Muller Medical College for his support.

\section{References}

1. Adams L. Managing side effects in radiation therapy patients. Radiation Therapist 2009; 18:109-23.

2. Tabbara, K. F. Tears. In: Vaughan, D., Asbury, T., Tabbara, K. F., eds. General ophthalmology, 12 th ed. East Norwalk, CT: Appleton \& Lange; 1989:67-73.

3. Scheie, H. G.; Albert, D. M., eds. Textbook of ophthalmology, 9th ed. Philadelphia: WB Saunders; 1977:50

4. Bessell EM, Henk JM, Whitelocke RA, et al. Ocular morbidity after radiotherapy of orbital and conjunctival lymphoma. Eye 1987; 1:90-96.

5. Brady LW et al. Ocular complications of high dose radiotherapy. Oncol 1996: 981-982

6. Heimann H, Coupland SE, GochmanR, Hellmich M and Foester MH. Alteration in expression of mucin, tenascin-c and syndecan-1 in the conjunctiva following retinal surgery and plaque radiotherapy. Graefes Arch Clin Exp Ophthalmol 2001; 488-495

7. Parsons JT, Bova FJ, Fitzgerald CR, et al. Severe dry-eye syndrome following external beam irradiation. Int J Radiat Oncol Biol Phys 1994; 30:775-780.

8. Bhandare N, Moiseenko V, Song WY, Morris CG, Bhatti MT, Mendenhall WM. Severe dry eye syndrome after radiotherapy for head and neck tumors. Int J Radiat Oncol Biol Phys. 2012; 82: 1501-1508 
9. Stephen LC et al. Radiation apoptosis of serous acinar cells of salivary and lacrimal glands. Cancer 1991: 1539-1543

10. The definition and classification of dry eye disease: Report of the Definition and Classification Subcommittee of the International Dry Eye WorkShop (2007). Ocul Surf 2007; 5:75-92

11. Bonomi AE, Palmers CS, Ajax M, Peeples P, Jackson SE: Cost of Man- aging mucositis and xerostomia in head and neck cancer patients undergoing chemoradiotherapy (CRT) or radiation (RT) [Abstract]. Value in Health 1999, 2:197.

12. Behrens A, Doyle JJ, Stern L, et al. Dysfunctional tear syndrome: A Delphi approach to treatment recommendations. Cornea 2006; 25:900-907.

13. Stafford SL et al. Orbital lymphoma: radiothearapy outcome and complications. Radiother Oncol $2001 ; 139-144$

Table 1: Dry Eye Severity Grading Scheme according to DEWS 2007

\begin{tabular}{|c|c|c|c|c|}
\hline $\begin{array}{l}\text { Dry Eye Severity } \\
\text { scale }\end{array}$ & Level 1 & Level 2 & Level 3 & Level 4\# \\
\hline $\begin{array}{l}\text { Discomfort, severity } \\
\text { and frequency }\end{array}$ & $\begin{array}{l}\text { Mild and/ } \\
\text { or episodic; } \\
\text { occurs under } \\
\text { environmental } \\
\text { stress }\end{array}$ & $\begin{array}{l}\text { Moderate } \\
\text { eoisodic or } \\
\text { chronic, stress } \\
\text { or no stress }\end{array}$ & $\begin{array}{l}\text { Severe frequent } \\
\text { or constant } \\
\text { without stress }\end{array}$ & $\begin{array}{l}\text { Severe and/or } \\
\text { disabling and } \\
\text { constant }\end{array}$ \\
\hline Visual symptoms & $\begin{array}{l}\text { None or } \\
\text { episodic mild } \\
\text { fatigue }\end{array}$ & $\begin{array}{l}\text { Annoying and/ } \\
\text { or activity } \\
\text { limiting, } \\
\text { episodic }\end{array}$ & $\begin{array}{l}\text { Annoying, } \\
\text { chronic and/ } \\
\text { or constant, } \\
\text { limiting activity }\end{array}$ & $\begin{array}{l}\text { Constant and/ } \\
\text { or possibly } \\
\text { disabling }\end{array}$ \\
\hline $\begin{array}{l}\text { Conjunctival } \\
\text { congestion }\end{array}$ & None to mild & None to mild & $+/-$ & $+/++$ \\
\hline $\begin{array}{l}\text { Conjunctival } \\
\text { staining }\end{array}$ & None to mild & Variable & $\begin{array}{l}\text { Moderate to } \\
\text { marked }\end{array}$ & Marked \\
\hline $\begin{array}{l}\text { Corneal } \\
\text { staining(severity/ } \\
\text { location) }\end{array}$ & None to mild & Variable & Marked central & $\begin{array}{l}\text { Severe } \\
\text { punctate } \\
\text { erosions }\end{array}$ \\
\hline Corneal/ tear sign & None to mild & $\begin{array}{l}\text { Mild debris, } \\
\text { reduced } \\
\text { meniscus } \\
\text { height }\end{array}$ & $\begin{array}{l}\text { Filamentary } \\
\text { keratitis, mucus } \\
\text { clumping, } \\
\text { increased tear } \\
\text { debris }\end{array}$ & $\begin{array}{l}\text { Filamentary } \\
\text { keratitis, mucus } \\
\text { clumping, } \\
\text { increased } \\
\text { tear debris, } \\
\text { ulceration }\end{array}$ \\
\hline $\begin{array}{l}\text { Lid/ meibomian } \\
\text { glands }\end{array}$ & $\begin{array}{l}\text { MGD variably } \\
\text { present }\end{array}$ & $\begin{array}{l}\text { MGD variably } \\
\text { present }\end{array}$ & Frequent & $\begin{array}{l}\text { Trichiasis, } \\
\text { keratinization, } \\
\text { symblepharon }\end{array}$ \\
\hline TFBUT(Second) & Variable & $\leq 10$ & $\leq 5$ & Immediate \\
\hline $\begin{array}{l}\text { Schirmer } \\
\text { test(mm/5min) }\end{array}$ & Variable & $\leq 10$ & $\leq 5$ & $\leq 2$ \\
\hline
\end{tabular}


TFBUT = Fluorescein Tear break-up time; $M G D=$ Meibomian gland disease \# Must have signs AND symptoms

Table 2: Patient and tumor characteristics

\begin{tabular}{|c|c|}
\hline Age & $50.3 \pm 8.57$ \\
\hline \multicolumn{2}{|l|}{ Gender } \\
\hline Male & 18 \\
\hline Female & 4 \\
\hline \multicolumn{2}{|l|}{ Site } \\
\hline Buccal mucosa & 6 \\
\hline Floor of the Mouth & 1 \\
\hline Gingivo buccal sulcus & 1 \\
\hline Lip & 1 \\
\hline Pharynx (Oro and Hypo) & 4 \\
\hline Retromolar trigone & 1 \\
\hline Supraglottis & 1 \\
\hline Tongue & 6 \\
\hline Vallecula & 1 \\
\hline \multicolumn{2}{|l|}{$\begin{array}{l}\text { TNM stage } \\
\text { Primary }\end{array}$} \\
\hline T1 & 0 \\
\hline T2 & 16 \\
\hline T3 & 4 \\
\hline T4 & 1 \\
\hline TX & 1 \\
\hline
\end{tabular}


Sowmya, Jayachander, Kamath, et al.

\begin{tabular}{|c|c|}
\hline \multicolumn{2}{|l|}{ Regional nodes } \\
\hline NO & 6 \\
\hline N1 & 5 \\
\hline N2 & 1 \\
\hline N2a & 1 \\
\hline N2b & 8 \\
\hline N2C & 1 \\
\hline \multicolumn{2}{|l|}{ Metastasis } \\
\hline MO & 22 \\
\hline MX & 0 \\
\hline \multicolumn{2}{|l|}{ Treatment details } \\
\hline Radiation only & 3 \\
\hline Chemoirradiation & 19 \\
\hline Dose of radiation & $68.2 \pm 1.98$ \\
\hline
\end{tabular}

